\title{
Treatment and management of Salmonella prostatitis in a heartworm-positive intact male dog: a case report
}

\author{
John N. Hertzer ${ }^{*}$, Madeline Fujishiro², Sara D. Lawhon ${ }^{3}$ and Kate E. Creevy ${ }^{1}$
}

\begin{abstract}
Background: Salmonella spp. represent a significant zoonotic concern to pregnant owners as infection can cause septic abortions and post-partum illness. Enteric salmonellosis is well documented in canines however urinary salmonellosis is rarely described and Salmonella prostatitis has never been described in dogs.

Case presentation: This case report describes the diagnosis and management of a five-year-old, intact male Labrador Retriever mix dog that was diagnosed with Salmonella prostatitis among other comorbidities including heartworm infestation. Additionally, mitigation of zoonotic spread is emphasized as one of the owners was six months pregnant at the time of diagnosis.

Discussion: The pathogenesis of Salmonella prostatitis is unknown but explanations pertaining to enteric salmonellosis, such as the lifestyle and stress of living as a stray may have contributed and contamination from an enteric infection may have also been possible. Several recommendations were made to reduce the likelihood of zoonotic transmission including frequent hand washing, avoidance of the patient's mouth, change in location of where the patient was fed, the use of an isolated area outside for urination and defecation, and the use of dilute bleach to clean areas soiled by the patient's bodily fluids. Monitoring of the prostatic infection was facilitated with prostatic wash instead of urine culture. This decision was made as prostatic infections have been shown to intermittently shed bacteria into the urine, leading to possible false negative urine cultures and potential catastrophic zoonotic infection.
\end{abstract}

Keywords: Salmonella prostatitis, salmonella zoonosis, Zoonotic prevention

\section{Background}

Salmonella enterica is estimated to cause approximately 1.2 million human illnesses, 22,000 hospitalizations and 425 deaths annually in the United States, at an annual cost of $\$ 3.3$ billion [1-3]. In pregnant women, salmonellosis has been shown to cause septic abortion [4], post-abortion and post-partum septicemia [5], and neonatal septicemia [4]. Dogs are well-documented enteric hosts for Salmonella enterica with a prevalence

\footnotetext{
* Correspondence: Jnhertzer@gmail.com

'Department of Small Animal Clinical Sciences, Texas A\&M University, 77843-4474 College Station, TX, USA

Full list of author information is available at the end of the article
}

of $2.5 \%$, and are thus a potential source of zoonotic transmission [6-8]. Dogs are usually subclinical carriers and are less likely to develop clinically significant disease; however, they can shed the bacteria through feces intermittently for six weeks or more post infection [9]. Possible clinical signs associated with salmonellosis in dogs include anorexia, fever, or diarrhea with isolated reports of meningoencephalitis, diskospondylitis, and acute hepatic necrosis [7, 10-12]. Urinary salmonellosis, however, has rarely been described in dogs [13] and to the authors' knowledge, confirmed Salmonella spp. prostatitis has not been described in the dog. This case report describes the challenges associated with the

(C) The Author(s). 2021 Open Access This article is licensed under a Creative Commons Attribution 4.0 International License, which permits use, sharing, adaptation, distribution and reproduction in any medium or format, as long as you give appropriate credit to the original author(s) and the source, provide a link to the Creative Commons licence, and indicate if changes were made. The images or other third party material in this article are included in the article's Creative Commons licence, unless indicated otherwise in a credit line to the material. If material is not included in the article's Creative Commons licence and your intended use is not permitted by statutory regulation or exceeds the permitted use, you will need to obtain permission directly from the copyright holder. To view a copy of this licence, visit http://creativecommons.org/licenses/by/4.0/ The Creative Commons Public Domain Dedication waiver (http://creativecommons.org/publicdomain/zero/1.0/) applies to the data made available in this article, unless otherwise stated in a credit line to the data. 
management of a novel site of salmonellosis in a heartworm-positive intact male dog living with a pregnant owner.

\section{Case presentation}

A stray intact male Labrador Retriever mix, estimated to be five years old, was adopted and was promptly evaluated by a veterinarian for lethargy and poor appetite. Physical examination noted ectoparasitism and asymmetric left-sided facial muscle atrophy. Complete blood count $(\mathrm{CBC})$ revealed severe thrombocytopenia (0 platelets/microliter $[\mu \mathrm{L}]$, confirmed via blood smear) and chemistry profile revealed mild hyperglobulinemia (5.4 gram $[\mathrm{g}] /$ deciliter $[\mathrm{dL}])$. Urinalysis obtained via cystocentesis revealed pyuria with 6-20 white blood cells (WBC)/high power field (hpf). An in-house infectious disease ELISA ${ }^{1}$ was reported as heartworm antigen positive, Ehrlichia spp. antibody positive, and Anaplasma spp. antibody positive. Thoracic radiographs revealed a mild bronchointerstitial pulmonary pattern and mild pulmonary artery enlargement.

The dog was prescribed doxycycline at 5.7 milligrams (mg)/kilogram (kg) per os (PO) twice a day (BID) for 28 days, sulfamethoxazole and trimethoprim (SMZ-TMP) at $27.3 \mathrm{mg} / \mathrm{kg}$ PO once daily for 14 days, and prednisone at $1.1 \mathrm{mg} / \mathrm{kg}$ PO BID. He was also administered an anthelmintic and prescribed monthly heartworm, flea, and tick prevention.

At a two-week re-evaluation, his appetite had improved, and the owner reported he was polyuric and polydipsic. $\mathrm{CBC}$ revealed an improvement in his thrombocytopenia $(156,000$ platelets/ $\mu \mathrm{L})$ and his prednisone dose was tapered by $25 \%$. At his recheck three weeks later, his urine was reported to be foul-smelling. His CBC revealed a worsening thrombocytopenia (117, 000 platelets $/ \mu \mathrm{L}$ ) and chemistry profile was unremarkable. Urinalysis reported pyuria and rod-shaped bacteria. Urine obtained via cystocentesis was submitted for aerobic culture and amoxicillin/clavulanic acid ${ }^{2}$ was prescribed at $13.8 \mathrm{mg} / \mathrm{kg}$ PO BID while results were pending. Due to his progressive thrombocytopenia, his prednisone was returned to the $1.1 \mathrm{mg} / \mathrm{kg}$ PO BID dose and he was prescribed azathioprine at $2.4 \mathrm{mg} / \mathrm{kg} \mathrm{PO}$ once daily. Urine culture results reported $>100,000$ organisms/milliliter $(\mathrm{mL})$ of Salmonella spp. and he was prescribed amoxicillin $25.3 \mathrm{mg} / \mathrm{kg}$ PO BID for 28 days (amoxicillin/clavulanic acid was discontinued). Ten days later, he was evaluated for a three-day history of progressive unilateral (left-sided) epistaxis and observed "red urine". CBC revealed a severe thrombocytopenia $(23,000$ platelets $/ \mu \mathrm{L}$ ); clotting times (prothrombin time and

\footnotetext{
${ }^{1}$ 4Dx SNAP test; Idexx, Inc., Westport ME.

${ }^{2}$ Clavamox, Zoetis Petcare, Parsippany-Troy Hills, NJ.
}

partial thromboplastin time) were within normal limits. $\mathrm{He}$ was referred to the Texas A\&M University Veterinary Medical Teaching Hospital (TAMU-VMTH) for further evaluation.

At TAMU-VMTH six weeks after original adoption, problems and considerations for this patient have been summarized in Table 1 and included:

- heartworm infestation (doxycycline had been begun prior to adulticide therapy).

- Ehrlichia spp. and Anaplasma spp. seropositivity (doxycycline had been begun).

- intermittent epistaxis and gross hematuria (possibly resulting from systemic infectious or inflammatory vasculitis or thrombocytopenia, or from two local diseases).

- varying thrombocytopenia (while only one measured value was severely low, the presence of clinical bleeding increased suspicion of true severity; immunosuppression with prednisone and azathioprine had been begun).

- facial asymmetry (relationship with concurrent epistaxis was unclear).

Salmonella culture-positive urine (concurrent hematuria; in an intact male dog, complicating prostatitis was presumed; poor anesthetic candidate for castration until heartworm adulticidal therapy was complete).

At the time of the TAMU-VMTH visit, it was also revealed that one of the owners was approximately six months pregnant with the couple's first child, raising additional concerns regarding treatment and precautions of the diagnosed Salmonella infection. Once the diagnosis of salmonellosis was determined, the owners were instructed to have the dog urinate/defecate in an isolated portion of the yard that the other housemate, a 10-yearold castrated male German Shepherd, could not access. The male owner was instructed to remove the feces while wearing gloves. If any urination or defecation occurred within the house, the male owner was instructed to use gloves while removing organic material and to then clean the area with a bleach-based solution. It was recommended to move the patient's water and food bowls to a separate, low-traffic area of the house to minimize Salmonella exposure in the kitchen. Both owners were also instructed to wash their hands frequently.

Static asymmetric left-sided facial muscle atrophy and pain on prostatic palpation were found. $C B C$ revealed 171,000 platelets/ $\mu \mathrm{L}$ and chemistry profile reported severe elevations in liver enzymes suspected to be secondary to azathioprine hepatotoxicity. Blood pressure was normal and thoracic radiographs confirmed 
Table 1 Summary of clinical problems with associated action steps

\begin{tabular}{|c|c|c|}
\hline Clinical Finding & Source & Action \\
\hline \multicolumn{3}{|l|}{ Thrombocytopenia } \\
\hline 0 plt/ $\mu \mathrm{L}$ & RDVM & Begin prednisone 2.2 mg/kg/day PO \\
\hline $156,000 \mathrm{plt} / \mu \mathrm{L}$ & RDVM & Decrease prednisone by $25 \%$ \\
\hline $117,000 \mathrm{plt} / \mu \mathrm{L}$ & RDVM & $\begin{array}{l}\text { Increase prednisone to } 2.2 \mathrm{mg} / \mathrm{kg} / \text { day PO; } \\
\text { begin azathioprine } 2.4 \mathrm{mg} / \mathrm{kg} \text { PO daily }\end{array}$ \\
\hline $23,000 \mathrm{plt} / \mu \mathrm{L}$ & RDVM & Refer to TAMU-VMTH \\
\hline $171,000 \mathrm{plt} / \mu \mathrm{L}$ & $\begin{array}{l}\text { TAMU- } \\
\text { VMTH }\end{array}$ & $\begin{array}{l}\text { Discontinue azathioprine; } \\
\text { decrease prednisone by } 50 \%\end{array}$ \\
\hline $102,000 \mathrm{plt} / \mu \mathrm{L}$ & $\begin{array}{l}\text { TAMU- } \\
\text { VMTH }\end{array}$ & $\begin{array}{l}\text { Begin cyclosporine at } 4.7 \mathrm{mg} / \mathrm{kg} / \mathrm{day} \mathrm{PO} ; \\
\text { begin Yunnan Baiyao } 0.5 \mathrm{~g} \mathrm{PO} \mathrm{BID;} \\
\text { increase prednisone by } 25 \%\end{array}$ \\
\hline $160,000 \mathrm{plt} / \mu \mathrm{L}$ & $\begin{array}{l}\text { TAMU- } \\
\text { VMTH }\end{array}$ & $\begin{array}{l}\text { Taper cyclosporine to discontinuation; } \\
\text { discontinue Yunnan Baiyao }\end{array}$ \\
\hline \multicolumn{3}{|l|}{ Vector-borne diseases } \\
\hline Ehrlichia spp. antibody seropositivity & RDVM & Begin doxycycline 5.7 mg/kg PO BID \\
\hline Anaplasma spp. antibody seropositivity & RDVM & Begin doxycycline 5.7 mg/kg PO BID \\
\hline D. immitis antigen seropositivity & RDVM & Begin doxycycline 5.7 mg/kg PO BID \\
\hline Echocardiographic confirmation of heartworm infestation & $\begin{array}{l}\text { TAMU- } \\
\text { VMTH }\end{array}$ & Administer melarsomine three-dose protocol (2.5 mg/kg/dose) \\
\hline \multicolumn{3}{|l|}{ Urinary tract infection } \\
\hline 6-20 WBC/hpf on UA & RDVM & $\begin{array}{l}\text { Begin empiric sulfamethoxazole- trimethoprim } 27.3 \mathrm{mg} / \mathrm{kg} P O \\
\text { daily } \times 14 \mathrm{~d}\end{array}$ \\
\hline $\begin{array}{l}\text { bacteriuria / pyuria } \\
\text { on UA }\end{array}$ & RDVM & Begin empiric amoxicillin/clavulanic acid 13.8 mg/kg PO BID \\
\hline$>100,000$ organisms/mL of Salmonella spp. from urine culture & RDVM & $\begin{array}{l}\text { Discontinue amoxicillin/clavulanic acid; } \\
\text { begin amoxicillin } 25.3 \mathrm{mg} / \mathrm{kg} \text { PO BID x 28d }\end{array}$ \\
\hline $\begin{array}{l}4 \text { colonies of Salmonella spp. from prostatic wash culture and } \\
\text { susceptibility }\end{array}$ & $\begin{array}{l}\text { TAMU- } \\
\text { VMTH }\end{array}$ & $\begin{array}{l}\text { Begin enrofloxacin } 10.6 \mathrm{mg} / \mathrm{kg} \text { PO daily x 28d; } \\
\text { begin doxycycline } 10.1 \mathrm{mg} / \mathrm{kg} \text { PO BID } \times 28 \mathrm{~d} \text {; } \\
\text { initiate zoonosis precautions in the home }\end{array}$ \\
\hline Negative urine culture after 14 days of enrofloxacin / doxycycline & $\begin{array}{l}\text { TAMU- } \\
\text { VMTH }\end{array}$ & No action \\
\hline $\begin{array}{l}\text { > 100,000 organisms/mL of Salmonella spp. from prostatic wash } \\
\text { culture }\end{array}$ & $\begin{array}{l}\text { TAMU- } \\
\text { VMTH }\end{array}$ & $\begin{array}{l}\text { Begin marbofloxacin } 3 \text { mg/kg PO daily through } 2 \text { weeks post- } \\
\text { castration }\end{array}$ \\
\hline Negative prostatic wash culture $10 \mathrm{~d}$ after stopping marbofloxacin & $\begin{array}{l}\text { TAMU- } \\
\text { VMTH }\end{array}$ & \\
\hline
\end{tabular}

Clinical data and action steps are in chronological order within each problem category

RDVM referring veterinarian. TAMU-VMTH Texas A\&M University Veterinary Medical Teaching Hospital

previous findings consistent with heartworm infestation. Abdominal ultrasound revealed a few small ill-defined hypoechoic liver nodules (suspect vacuolar hepatopathy), slightly small adrenal glands bilaterally (likely secondary to exogenous corticosteroids), and a mildly enlarged irregular heterogenous prostate (suspect prostatitis). An echocardiogram was performed and identified one heartworm in the distal right pulmonary artery with no significant cardiac remodeling or evidence of pulmonary hypertension. A prostatic wash cytology showed mild atypical epithelial cells (suspect reactive change) and culture of prostatic wash fluid isolated four colonies of Salmonella species. The isolate was initially identified as Salmonella species using matrix-assisted laser desorption-time of flight (MALDI-TOF) mass spectrometry using the Biotyper ${ }^{\mathrm{rm} 3}$ with flexControl v3.4 build 135.14 software. The isolate from this culture was submitted to the National Veterinary Service Laboratories (NVSL) in Ames, Iowa for serotyping and was determined to be Salmonella enterica serovar III 41:z4,z23:Subsequently the isolate was sequenced using Illumina MiSeq and confirmed to be Salmonella enterica subsp. arizonae serovar 41:z4,z23:-. The isolate was then sequenced using $2 \times 300$ bp paired-end reads $^{4}$ and confirmed to be Salmonella enterica subsp. arizonae

\footnotetext{
$\overline{{ }^{3} \text { Bruker Daltonics, Billerica, MA] }}$

${ }^{4} \mathrm{MiSeq}^{\mathrm{Tn}}$, Illumina, San Diego, CA.
} 
serovar 41:z4,z23:- using SeqSero, in agreement with the NVSL report [14]. Details of the sequence analysis have been previously published [15]. Based on culture and susceptibility results, enrofloxacin at $10.6 \mathrm{mg} / \mathrm{kg} \mathrm{PO}$ once daily for 28 days was prescribed, as well as another course of doxycycline at $10.1 \mathrm{mg} / \mathrm{kg}$ PO BID for 28 days and reduction of the prednisone dose $1.0 \mathrm{mg} / \mathrm{kg} \mathrm{PO}$ once daily. Azathioprine and amoxicillin were discontinued.

Magnetic resonance imaging (MRI) and computed tomography $(\mathrm{CT})$ of the brain/skull performed one week later did not reveal a cause for the dog's facial asymmetry and left-sided epistaxis. Cerebrospinal fluid (CSF) analysis revealed no cytological abnormalities. A von Willebrand factor antigen assay (vWF:Ag) returned a result of $33 \%$, with $<50 \%$ being consistent with an abnormal result or carrier range. Yunnan Baiyao at $0.5 \mathrm{~g}$ PO BID was prescribed. Because CBC revealed progressive thrombocytopenia $(102,000$ platelets $/ \mu \mathrm{L})$, cyclosporine at $4.7 \mathrm{mg} / \mathrm{kg} \mathrm{PO}$ once daily was prescribed and prednisone was increased to $1.25 \mathrm{mg} / \mathrm{kg}$ PO BID. No further episodes of epistaxis were reported, and Yunnan Baiyao was eventually discontinued without any adverse bleeding events.

Two weeks after initiating enrofloxacin, urine collected by cystocentesis yielded no bacterial growth on quantified urine culture. CBC revealed 160,000 platelets $/ \mu \mathrm{L}$ and chemistry profile showed moderate improvement of the hepatopathy. Immune-mediated platelet destruction was considered less likely at this point due to resolution of bleeding events, and persistence of only moderate, fluctuating thrombocytopenia. Cyclosporine was slowly tapered without a relapse of severe thrombocytopenia and prednisone was tapered once heartworm adulticide therapy was completed. The platelet count remained mildly low throughout treatment for heartworm disease and Salmonella prostatitis. An underlying etiology was never confirmed.

Approximately one month later when the liver enzyme elevation had resolved, the dog returned for the first heartworm adulticide treatment with melarsomine at $2.5 \mathrm{mg} / \mathrm{kg}$ as recommended by the American Heartworm Society [16]. A prostatic wash culture was also performed (two weeks after completion of enrofloxacin). The culture grew $>100,000$ organisms $/ \mathrm{mL}$ of Salmonella spp. It was unknown if the prostate had remained persistently infected (as only a urine culture without prostate wash had been performed at midtreatment recheck) or if repeated re-infections of the prostate were occurring. The isolate was submitted to NVSL and WGS was performed and the serotype was the same as that previously isolated from the patient's urine (serotype 41:z4,z23:-). Fecal Salmonella PCR to detect the spaQ was performed on three fecal samples collected $24 \mathrm{~h}$ apart and all three samples were positive. An isolate recovered from the feces was submitted to NVSL and determined to be Salmonella enterica subsp arizonae serovar 41:z4,z23:-, the same serovar isolated from the prostate. The PCR was performed as previously described [17] with the modifications that the PCR was performed using a different real time PCR thermocycler ${ }^{5}$ and PCR reagents. ${ }^{6} \mathrm{~A}$ positive control of genomic DNA from a clinical strain of Salmonella enterica subspecies enterica serovar Typhimurium was utilized. Negative control PCR reactions included reactions with either genomic DNA from Escherichia coli strain ATCC 29,522 or with no DNA added to the reaction. Identification was confirmed with serotyping by the National Veterinary Services Laboratory.

Due to the challenge of curing bacterial prostatitis in an intact male dog, castration was planned as soon as anesthesia could safely be performed after heartworm adulticide therapy. Based on culture and sensitivity results, and considerations for adequate prostatic and fecal penetration, marbofloxacin was prescribed at $3 \mathrm{mg} /$ $\mathrm{kg} \mathrm{PO}$ once daily. In an attempt to decrease environmental contamination of the home environment with Salmonella, antimicrobial therapy was planned for the duration of his heartworm therapy. Heartworm therapy with two additional doses of melarsomine was completed six weeks later, after delivery of the owners' new baby. A prostatic wash culture was performed, and Salmonella was isolated after broth enrichment (serotype 41:z4,z23:-). Fecal Salmonella PCR was also performed and was negative on all three samples. Marbofloxacin was continued.

One month later when the dog was considered a more stable anesthetic candidate, castration was performed. Desmopressin $0.1 \mathrm{mg}$ was administered subcutaneously $30 \mathrm{~min}$ prior to surgery due to the low vWF:Ag and surgery was completed without incident. A prostatic wash culture wash also performed and reported no growth. Marbofloxacin was continued for two additional weeks. Ten days after stopping marbofloxacin, a final prostatic wash culture was performed and reported no growth.

\section{Discussion and conclusions}

In this case, the source of the Salmonella infection is unknown. Several lifestyle factors of this patient suggest possible sources. First, the presumed predatory and scavenger lifestyle while living as a stray may have increased the exposure risk to Salmonella. Salmonella enterica subspecies arizonae is a commensal organism in reptiles,

\footnotetext{
${ }^{5}$ ABI 7900HT Fast real time PCR machine (Applied Biosystems)]

${ }^{6}$ Taqman Fast Universal PCR Master Mix 2X, No Amperase UNG, ThermoFisher Scientific Waltham, MA.
} 
such as snakes, and has been associated with disease in poultry $[18,19]$. It is possible that this patient had prior exposure to or ingestion of reptiles while living as a stray. It has been reported that dogs in Texas animal shelters had an overall prevalence of fecal Salmonella of $4.9 \%$, and the rate tended to be higher among dogs who arrived at the shelter as strays than among those who had been surrendered; importantly, Salmonella-positive feces in this study were most often normal in appearance [20]. Additionally, the physiologic stress of living as a stray may have made this patient more susceptible to infection. Chronic stress increases exposure to catecholamines and glucocorticoids, which can influence humoral immunity as well as intestinal flora growth, biofilm formation, and motility, potentially allowing for pathogenic bacterial colonization within the intestines $[20,21]$. These explanations are commonly applied to enteric salmonellosis. The pathogenesis of this patient's Salmonella prostatitis is unknown, but it is plausible that the same risks of exposure and impaired immune clearance are relevant to salmonellosis outside the gastrointestinal tract as well. It is also plausible that this patient's urinary tract infection was a result of contamination from an original enteric infection.

Castration is generally recommended as adjunct treatment for prostatitis in dogs [22-24]. In the prospective study by Cowan et al. [24], castration reduced the period of positive prostatic culture by more than four weeks. Additionally, castration was also shown in that study to significantly reduce the amount of bacteria isolated from the urine when compared to intact males [24]. With these facts in mind, it was imperative in this case to castrate the patient as soon as possible to help prevent zoonotic spread of the isolated Salmonella. Until very recently, canine castration has only been described while under general anesthesia mainly due to the large amount of perceived nociception associated with the procedure [25]. In non-shelter settings, however, heartworminfested patients are considered to be at increased risk of anesthetic complications [26]. This increased risk is partly due to the reduction in pulmonary vascular resistance, which can result in post-operative pulmonary thromboembolism [26]. Because of this increased risk for this patient, castration was scheduled after completion of heartworm adulticidal therapy.

Regardless of when castration would occur, it was imperative to implement a protocol at home to help prevent zoonotic spread to the pregnant owner. The first recommendation was to have the non-pregnant owner take over feeding of the patient. Additionally, frequent hand washing was recommended for both owners, especially after interacting with the patient in any way and prior to eating a meal [27]. If the pregnant owner were to touch any of the patient's bodily fluids, it was recommended to wear gloves followed by careful hand washing. Avoidance of the patient's mouth was also recommended to prevent contact with saliva [27]. The owners were also asked to feed the patient in a different room than the kitchen to reduce the amount of time the patient spent in areas where preparation and consumption of human food occurred. In a recent Salmonella outbreak in children that was associated with pet food, one of the identified risk factors pertained to where pets were fed [28]. When outside, it was recommended to have the patient urinate and defecate in a separate, isolated portion of the yard not used by other pets, and where the non-pregnant owner could promptly remove any feces; as mentioned previously, even non-diarrheic canine feces can be positive for Salmonella [20, 29]. Lastly, when the non-pregnant owner needed to clean an area soiled by the patient's bodily fluids, disinfection with household bleach, diluted 1:10 to 1:20 with water, was recommended after any organic material had been removed [29].

In this case, it was important to monitor infection status until clearance of Salmonella could be confirmed. Options for monitoring included urine culture and fecal PCR, as well as prostatic wash cultures. Prostatic wash was chosen for several reasons. It has been shown that prostatic infections can intermittently shed bacteria into the urine, leading to false negatives if urine culture alone were used [24]. In this case, a false negative urine culture at the ten-day post-antibiotic recheck would have increased the risk of potentially catastrophic zoonosis. In regards to fecal PCR, currently it is recommended that if a PCR-positive result for Salmonella is obtained, a fecal culture should be performed to identify the organism [29]. In this case, organism identification by fecal culture following a positive fecal PCR was considered to be less clinically relevant than identification of the organism in the urinary tract. Similarly, it was not clear that a negative fecal PCR would indicate a concurrent clearance of the urinary infection. Confirmed Salmonella spp. prostatitis has not previously been described in the dog. In the dog of this case, treatment of heartworm disease, followed by castration, facilitated successful resolution of Salmonella prostatitis in a dog living with a pregnant owner. This report highlights the importance of using prostatic wash to monitor prostatic infection as intermittent bacteriuria was observed. Additionally, this report provides a practical review on the importance and logistics of reducing the risk of zoonotic infection for dog owners.

\section{Abbreviations}

Spp.: Species, plural; CBC: Complete blood count; WBC: White blood cells: ELISA: Enzyme-linked immunoassay; PO: per os (by mouth); BID: bis in die (twice a day); TAMU-VMTH: Texas A\&M University Veterinary Medical Teaching Hospital; MALDI-TOF: Matrix-assisted laser desorption-time of 
flight.; NVSL: National Veterinary Science Laboratory; MRI: Magnetic resonance imaging; CT: Computed tomography; VWF:Ag: :Von Willebrand Factor antigen assay; WGS: Whole genome sequencing; PCR: Polymerase chain reaction

\section{Acknowledgements}

Not applicable.

\section{Authors' contributions}

$\mathrm{KC}$ and MF were the primary clinicians on the case and SL was the microbiologist involved with the case. JH and MF wrote the manuscript. All authors reviewed the manuscript. All authors read and approved the final manuscript.

\section{Funding}

None.

\section{Availability of data and materials}

All data generated or analyzed during this study are included in this published article.

\section{Declarations}

\section{Ethics approval and consent to participate}

Not applicable.

\section{Consent for publication}

Written consent for publication was obtained from the patient's owner

\section{Competing interests}

The authors declare that they have no competing interests.

\section{Author details}

'Department of Small Animal Clinical Sciences, Texas A\&M University, 77843-4474 College Station, TX, USA. ${ }^{2}$ Las Vegas Veterinary Specialty Center, 89147 Las Vegas, NV, USA. ${ }^{3}$ Department of Veterinary Pathobiology, Texas A\&M University, 77843-4467 College Station, TX, USA.

Received: 14 December 2020 Accepted: 14 March 2021

\section{Published online: 30 March 2021}

\section{References}

1. Hale CR, Scallan E, Cronquist AB, Dunn J, Smith K, Robinson T, et al. Estimates of enteric illness attributable to contact with animals and their environments in the United States. Clin Infect Dis. 2012;54(suppl_5):472-S9.

2. Hoffmann S, Batz MB, Morris JG. JR. Annual cost of illness and qualityadjusted life year losses in the United States due to 14 foodborne pathogenst. J Food Prot. 2012:75(7):1292-302

3. Scallan E, Hoekstra RM, Angulo FJ, Tauxe RV, Widdowson MA, Roy SL, et al. Foodborne illness acquired in the United States-major pathogens. Emerg Infect Dis. 2011;17(1):7-15.

4. Scialli $A R$, Rarick TL. Salmonella sepsis and second-trimester pregnancy loss. Obstet Gynecol. 1992;79(5 (Pt 2)):820-1.

5. van der Klooster JM, Roelofs HJ. Management of Salmonella infections during pregnancy and puerperium. Neth J Med. 1997:51(2):83-6.

6. Moran AB, editor. Occurrence and distribution of Salmonella. in animals in the United States. Proceedings, 65th annual meeting, US Livestock Sanitary Association, Minneapolis, Minn; 1961.

7. Leonard F. Salmonella infection and carriage: the importance of dogs and their owners. Vet Rec. 2014;174:92-3.

8. Cummings KJ, Mitchell PK, Rodriguez-Rivera LD, Goodman LB. Sequence analysis of Salmonella enterica isolates obtained from shelter dogs throughout Texas. Vet Med Sci. 2020.

9. Finley R, Ribble C, Aramini J, Vandermeer M, Popa M, Litman M, et al. The risk of salmonellae shedding by dogs fed Salmonella-contaminated commercial raw food diets. Can Vet J. 2007:48(1):69-75.

10. Plessas IN, Jull P, Volk HA. A case of canine discospondylitis and epidural empyema due to Salmonella species. Can Vet J. 2013:54(6):595-8.

11. Giuliano A, Meiring T, Grant AJ, Watson PJ. Acute Hepatic Necrosis Caused by Salmonella enterica Serotype | 4,5,12:-:1,2 in a Dog. J Clin Microbiol. 2015; 53(11):3674-6
12. Andruzzi MN, Krath ML, Lawhon SD, Boudreau B. Salmonella enterica subspecies houtenae as an opportunistic pathogen in a case of meningoencephalomyelitis and bacteriuria in a dog. BMC Vet Res. 2020; 16(1):437.

13. Cole SD, Palermo SM, Rankin SC. Salmonella enterica serovar Typhimurium isolated from the urine of a dog undergoing treatment for immunemediated polyarthritis. JMM Case Rep. 52018. p. e005153.

14. Zhang S, Yin Y, Jones MB, Zhang Z, Deatherage Kaiser BL, Dinsmore BA, et al. Salmonella serotype determination utilizing high-throughput genome sequencing data. J Clin Microbiol. 2015;53(5):1685-92.

15. Krath ML, Little SV, Hillhouse AE, Lawhon SD. Salmonella enterica subsp. arizonae Isolated from a canine clinical case of prostatitis. Microbiol Resour Announc. 2020;9(13).

16. Nelson CT, McCall JW, Stephen Jones S, Moorhead A. Prevention, diagnosis, and danagement of heartworm (Dirofilaria immitis) infection in dogs. American Heartworm Society 2020. https://www.heartwormsociety.org/ veterinary-resources/american-heartworm-society-guidelines. Accessed 1 Dec 2020.

17. Kurowski PB, Traub-Dargatz JL, Morley PS, Gentry-Weeks CR. Detection of Salmonella spp in fecal specimens by use of real-time polymerase chain reaction assay. Am J Vet Res. 2002;63(9):1265-8.

18. Tracy LM, Hicks JA, Grogan KB, Nicholds JA, Morningstar-Shaw BR, Shariat NW. Molecular detection of Salmonella enterica subsp. arizonae by quantitative PCR. Avian Dis. 2020;64(3):305-9.

19. Hopkins KL, Peters TM, Lawson AJ, Owen RJ. Rapid identification of Salmonella enterica subsp. arizonae and S. enterica subsp. diarizonae by realtime polymerase chain reaction. Diagn Microbiol Infect Dis. 2009;64(4):452-4.

20. Leahy AM, Cummings KJ, Rodriguez-Rivera LD, Rankin SC, Hamer SA. Evaluation of faecal Salmonella shedding among dogs at seven animal shelters across Texas. Zoonoses Public Health. 2016;63(7):515-21.

21. Verbrugghe E, Boyen F, Gaastra W, Bekhuis L, Leyman B, Van Parys A, et al. The complex interplay between stress and bacterial infections in animals. Vet Microbiol. 2012;155(2-4):115-27.

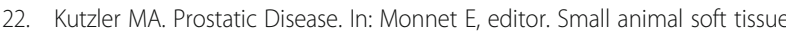
surgery. Hoboken: Wiley Online Library; 2012. pp. 667-80.

23. Smith J. Canine prostatic disease: a review of anatomy, pathology, diagnosis, and treatment. Theriogenology. 2008;70(3):375-83.

24. Cowan LA, Barsanti JA, Crowell W, Brown J. Effects of castration on chronic bacterial prostatitis in dogs. J Am Vet Med Assoc. 1991;199(3):346-50.

25. Silva E, Schumacher J, Passler T. Castration of dogs using local anesthesia after sedating with xylazine and subanesthetic doses of ketamine. Front Vet Sci. 2019:6:478.

26. Peterson KM, Chappell DE, Lewis B, Staton A, Dement E, Prater PE, et al. Heartworm-positive dogs recover without complications from surgical sterilization using cardiovascular sparing anesthesia protocol. Vet Parasitol. 2014:206(1-2):83-5.

27. Robinson RA, Pugh N, Dogs R. zoonoses immunosuppressionJ R Soc Promot Health. 2002;122(2):95-8.

28. Behravesh CB, Ferraro A, Deasy M, Dato V, Moll M, Sandt C, et al. Human Salmonella infections linked to contaminated dry dog and cat food, 20062008. Pediatrics. 2010;126(3):477-83.

29. Marks SL, Rankin SC, Byrne BA, Weese JS. Enteropathogenic bacteria in dogs and cats: diagnosis, epidemiology, treatment, and control. J Vet Intern Med. 2011;25(6):1195-208

\section{Publisher's Note}

Springer Nature remains neutral with regard to jurisdictional claims in published maps and institutional affiliations. 